PROCEEDINGS OF THE

AMERICAN MATHEMATICAL SOCIETY

Volume 132, Number 6, Pages 1805-1822

S 0002-9939(04)07272-7

Article electronically published on January 7, 2004

\title{
INSTABILITY OF STATISTICAL FACTOR ANALYSIS
}

\author{
STEVEN P. ELLIS
}

(Communicated by Richard A. Davis)

\begin{abstract}
Factor analysis, a popular method for interpreting multivariate data, models the covariance among $p$ variables as being due to a small number $(k, 1 \leq k<p)$ of hidden variables. A factor analysis of $Y$ can be thought of as an ordered or unordered collection, $F(Y)$, of $k$ linearly independent lines in $\mathbb{R}^{p}$. Let $\mathcal{Y}^{\prime}$ be the collection of data sets for which $F(Y)$ is defined. The "singularities" of $F$ are those data sets, $Y$, in the closure, $\overline{\mathcal{Y}}^{\prime}$, at which the limit, $\lim _{Y^{\prime} \rightarrow Y, Y^{\prime} \in \mathcal{Y}^{\prime}} F\left(Y^{\prime}\right)$, does not exist. $F$ is unstable near its singularities.

Let $\Phi(Y)$ be the direct sum of the lines in $F(Y)$. $\Phi$ determines a $k$-plane bundle, $\eta$, over a subset, $\mathcal{X}$, of $\mathcal{Y}$. If $k>1$ and $\eta$ is rich enough, $F$ ordered or, at least if $k=2$ or 3 , unordered, must have a singularity at some data set in $\mathcal{X}$. The proofs are applications of algebraic topology. Examples are provided.
\end{abstract}

\section{INTRODUCTION}

Factor analysis, a popular statistical method for simplifying and interpreting multivariate data, approximates the covariance (or correlation) among a collection of variables by the covariance of linear combinations of hidden "common" and "specific" variables ("factors"). The number of common factors is usually assumed to be small, so that the result is a simpler, and, hopefully, interpretable description of the dependence among the original variables. For an example of the use of factor analysis, see [19, pp. 102-115]. For a discussion of factor analysis as a form of "representational learning", see [5, Section 10.3]. 12 is an important reference on factor analysis and reviews its history. 15 is a more mathematical treatment. A number of different factor analysis methods have been proposed ([12, 15]). One is described in this section and another in section 7 In this paper we use topological methods to show that under mild hypotheses on a factor analysis method, $F$, there will exist at least one data set near which $F$ is unstable, i.e., highly sensitive to small changes in the data.

Let

$$
1<k<p<n
$$

be integers. Let $y_{1}, \ldots, y_{n} \in \mathbb{R}^{p}$ be "the data" ( $\mathbb{R}=$ reals). Each $y_{i}$ consists of the values of $p$ quantitative variables measured on a single unit of observation

Received by the editors December 3, 2001.

2000 Mathematics Subject Classification. Primary 62H25; Secondary 65D10.

Key words and phrases. Vector bundle, maximum likelihood, principal components.

This research is supported in part by United States PHS grants MH46745, MH60995, and MH62185. 
(human subject, say). Thus, each coordinate axis in $\mathbb{R}^{p}$ corresponds to a variable. Let $Y^{n \times p}$ be the matrix whose $i^{\text {th }}$ row is $y_{i}$. (We commonly use superscripts to indicate matrix dimensions.) Let $\mathcal{Y}$ denote the set of all $n \times p$ real matrices. $\mathcal{Y}$ is homeomorphic to $\mathbb{R}^{n p}$. In factor analysis one tries to find matrices $K^{p \times k}=K(Y)$, $C^{k \times k}=C(Y)$, and $D^{p \times p}=D(Y)$ such that (s.t.) $K$ has rank $k, C$ is symmetric and positive definite ([15, Section 2.5]), $D$ is diagonal with nonnegative entries, and, if $S=S(Y)$ is the sample covariance matrix of $Y$ (see (7.6) for definition), then

$$
S=K C K^{T}+D,
$$

at least approximately ([15, Section 2.5]). (Here, "T" indicates transposition.) Alternatively, the correlation matrix can be used instead of $S . K$ is called the "factor loading matrix". Call its columns "loading vectors". The diagonal elements of $D$ are the "specific variances".

Equation (1.2) does not have a unique solution: If $K$ is a solution and $R^{k \times k}=$ $R(Y)$ is invertible, then $S=(K R)\left(R^{-1} C\left(R^{T}\right)^{-1}\right)(K R)^{T}+D$ is also a solution. In particular, $C$ is arbitrary and often is constrained to be $I_{k}$, the $k \times k$ identity matrix (e.g., in section 7). However, in general $C$ depends on $Y$. The operation $K \mapsto K R$ is called "rotation" and is used in hopes of making the loading vectors easier to interpret, which happens, roughly speaking, when they are nearly parallel to coordinate planes in $\mathbb{R}^{p}$. It is important to note that rotation does not affect the column space of the factor loading matrix. If (1.2) holds exactly, we say that (1.2) is a "perfect fit" to $Y$ or, for short, " $Y$ is a perfect fit."

The matrix $K$ is the most important component of the analysis. However, for the purpose of interpreting $K$, factor analysts usually ignore some of its structure. For example, the interpretation of $K$ is unchanged if any of its columns are replaced by nonzero multiples. So it makes sense to think of the results of the factor analysis as being the lines spanned by the individual loading vectors. Call these lines the "factor lines". Call factor analysis, thought of as a function taking data, $Y$, to an (ordered) $k$-tuple, $F_{(k)}(Y)$, of lines in $\mathbb{R}^{p}$, "ordered factor analysis".

However, often even less structure than that is used. It is common to specify a loading vector not by its position in the matrix, but by some label depending only on the coordinates of the vector. For example, if a loading vector in a factor analysis of IQ test data is nearly parallel to a coordinate plane spanned by variables all measuring aspects of verbal ability, it might be thought of as representing a "Verbal Comprehension Factor" ([19, Table 4.17, p. 105]). In this case, the loading vectors are unordered in the sense that no extrinsic labels for the loading vectors are used. Call factor analysis, thought of as a function taking data, $Y$, to an unordered collection, $F_{\{k\}}(Y)$, of $k$ lines in $\mathbb{R}^{p}$, "unordered factor analysis". Because we do not want to develop a theory depending on structures that might be ignored, until section 7 we will forget about (1.2) and just think of a factor analysis as a function $F_{(k)}$ or $F_{\{k\}}$. (In subsection 3.1 we will modify the definition to emphasize the role of the "factor space", defined presently.) Interpreting factor analysis as functions $F_{(k)}$ or $F_{\{k\}}$ appears not to be standard. However, besides adding more realism to the discussion, making $F_{(k)}$ and $F_{\{k\}}$ the objects of analysis leads to stronger results.

Jennrich [13] shows via algebraic and numerical examples that factor analysis can be unstable in the sense that small changes in the data can lead to large changes in the factor analysis. This paper is a general theoretical examination of one kind of 
instability of factor analysis. In general, $K(Y)$ - and, hence, $F_{(k)}(Y)$ and $F_{\{k\}}(Y)$ - will not be defined for every $Y \in \mathcal{Y}$. Let $\mathcal{Y}^{\prime}$ denote the set of $Y \in \mathcal{Y}$ for which $F_{(k)}(Y)$ is defined. $F_{(k)}$ is unstable in the vicinity of its "singularities," i.e., data sets $Y_{0}$ in the closure $\overline{\mathcal{Y}^{\prime}}$ s.t.

$$
\lim _{\substack{Y^{\prime} \rightarrow Y_{0}, Y^{\prime} \in \mathcal{Y}^{\prime}}} F_{(k)}\left(Y^{\prime}\right) \text { does not exist. }
$$

Singularity of unordered factor analysis is defined similarly. (Given the interpretation provided in subsection 3.1 the needed topologies are intuitive and defined in section 2.) It is reasonable to suppose that the probability of getting a data set in the vicinity of the singularities of $F_{(k)}$ is positive.

Factor analyses are not reproducible near singularities. That is, suppose that a data set, $Y$, is near a singularity of the factor analysis method used to analyze it. Suppose a similar but independent data set is gathered and the same factor analysis method is applied to the new data set. The results are unlikely to be like those from $Y$. Of course, such non-reproducible results can come about just by chance. Statistical methods can sometimes be used to estimate the probability of this happening. But non-reproducibility caused by singularity is of a different sort, because one can recognize when it has happened: In principle, one can check to see if one's data is near a singularity. Near its singularities a factor analysis method will also be sensitive to rounding error.

We separate out two aspects of factor analysis. Let $\Phi(Y)$ be the "factor space", i.e., the column space of $K(Y)$ (which is the same as the direct sum of the lines in $F_{(k)}$ or $\left.F_{\{k\}}\right) . \Phi$ is the "factor space function" of the factor analysis method. Note that $\Phi(Y)$ is unaffected by any factor rotations. The function $\Phi$ itself may have singularities similar to those of "plane-fitters" (6] 8]).

We focus on singularities of factor analysis that are not singularities of $\Phi$. Call these singularities "factor singularities". Let $\mathcal{X} \subset \mathcal{Y}$ be the set of points in $\overline{\mathcal{Y}^{\prime}}$ that are not singularities of $\Phi$. We may assume $\Phi$ is defined and continuous everywhere on $\mathcal{X}$. So $\Phi$ determines a $k$-plane bundle $([17]), \eta$, on $\mathcal{X}$. In subsection 3.3 we use the theory of characteristic classes ([17]) to show that, if $\eta$ is rich enough to contain a subbundle isomorphic to a small specific bundle, then $F_{(k)}$ must have a singularity at a data set in $\mathcal{X}$. Such a singularity is a factor singularity.

Note that the condition on $\eta$ will be satisfied if $\Phi$ often recognizes perfect fits, i.e., if for many data sets $Y$ for which (1.2) holds exactly, $\Phi(Y)$ is the obvious space - the column space of $K$. This is a natural property to expect of a factor analysis method, and is exemplified in section 7

As for unordered factor analysis, we show in section 6 that if $k=3$, then under the same hypotheses as in the ordered case, an unordered factor analysis method must have a factor singularity. This is done by reducing the unordered case to the ordered one. The $k=3$ result implies the $k=2$ result. I conjecture that the result holds for all $k>1$.

The hypotheses of our results only depend on $\Phi$. Thus, to check that the results apply to a particular factor analysis method, any factor rotations involved can be ignored. This is an advantage of our approach.

1.1. Example (Principal Components). Here is a simple but important example. Let $Y \in \mathcal{Y}$ and let $\lambda_{1} \geq \cdots \geq \lambda_{p} \geq 0$ be the eigenvectors of the covariance matrix, $S=S(Y)$. The first $k$ (sample) "principal components" of $Y$ based on $S$ are the 
$k$ (unit) eigenvectors, $v_{1}, \ldots, v_{k}$, of $S$ corresponding to $\lambda_{1} \geq \cdots \geq \lambda_{k}$, provided $\lambda_{1}>\cdots>\lambda_{k}>\lambda_{k+1}$. (Think of $v_{1}, \ldots, v_{k}$ as column vectors.) As a factor analysis method, "principal component analysis" (PC) corresponds to taking

$$
K=\left(\sqrt{\lambda_{1}} v_{1}, \ldots, \sqrt{\lambda_{k}} v_{k}\right) \text { and } C=I_{k}
$$

in (1.2). Since $D$ is not specified, strictly speaking $\mathrm{PC}$ is not a factor analysis method. However, it is very commonly considered one and, in any case, our results can be applied to it. Notice that the columns of $K$ are only determined up to sign. Since we only care about the lines spanned by the columns, this does not matter. The factor space, $\Phi(Y)$, is the column space of $K$ whenever it is defined. The singularities of $\Phi$ are precisely the data sets $Y$ s.t. $\lambda_{k}=\lambda_{k+1}$. The factor singularities of PC are precisely the data sets s.t. $\lambda_{k}>\lambda_{k+1}$ but $\lambda_{1}, \ldots, \lambda_{k}$ are not distinct. One might ask, can the factor singularities be eliminated by rotation? As an application of the main results of this paper we show in Example 7.1 that, at least if $1<k<p-2$ (and $k \leq 3$ in the unordered case), the answer is "no".

In section 2 definitions and basic theory of spaces of ordered and unordered collections of lines, and bundles of such spaces, are developed. In section 3, the existence of singularities for ordered factor analysis is proved under general assumptions. In sections 4 and 5 , the fundamental groups of the space of unordered collections of three orthogonal lines in $\mathbb{R}^{3}$ and the total space of a bundle of the same are computed. Section [6] shows that when $k=2$ or 3 , the existence of singularities for unordered factor analysis reduces to that for ordered factor analysis. Section 7 is devoted to examples. Section 8 is a brief summary and discussion of our results and their implications. The manuscript [7] is a detailed version of this paper.

\section{SPACES OF CROSSED LINES AND BUNDLES THEREOF}

2.1. Spaces of crossed lines. Factor analysis has to do with $k$ lines in $\mathbb{R}^{p}$, but as part of studying this we will have to consider the way $k$ lines sit in their $k$ dimensional direct sum. Let $\mathbf{L}_{(k)}$ denote the set of all $k$-tuples $\left(L_{1}, \ldots, L_{k}\right)$ of lines in $\mathbb{R}^{k}$ through the origin s.t. the direct sum of $L_{1}, \ldots, L_{k}$ is $\mathbb{R}^{k}$. $\mathbf{L}_{(k)}$ inherits its topology from $\left(P^{k-1}\right)^{k}$, where $P^{k-1}$ is $(k-1)$-dimensional projective space ([11. p. 25], [18, p. 231]). Let $\mathbf{J}_{(k)}$ denote the subset of $\mathbf{L}_{(k)}$ consisting of $k$-tuples of orthogonal lines.

Now consider the unordered case. Let $\mathbf{L}_{\{k\}}$ denote the set of all (unordered) sets, $\left\{L_{1}, \ldots, L_{k}\right\}$, of $k$ lines in $\mathbb{R}^{k}$ through the origin s.t. the direct sum of $L_{1}, \ldots, L_{k}$ is $\mathbb{R}^{k}$. Let $\rho$ be the projection that takes $\left(L_{1}, \ldots, L_{k}\right) \in \mathbf{L}_{(k)}$ to $\left\{L_{1}, \ldots, L_{k}\right\} \in \mathbf{L}_{\{k\}}$. Put on $\mathbf{L}_{\{k\}}$ the quotient topology ([18, pp. 111,112]) generated by $\rho$. Let $\mathbf{J}_{\{k\}}=$ $\rho\left(\mathbf{J}_{(k)}\right) \subset \mathbf{L}_{\{k\}}$ be the set of all unordered collections of $k$ orthogonal lines through the origin in $\mathbb{R}^{k}$. (The elements of $\mathbf{J}_{\{k\}}$ are "jacks".) $\mathbf{J}_{\{k\}}$ inherits its topology from $\mathbf{L}_{\{k\}}$. We have the following.

2.1. Proposition. There is a continuous map $r: \mathbf{L}_{(k)} \rightarrow \mathbf{J}_{(k)}$ with the following properties.

(1) If $J \in \mathbf{J}_{(k)}$, then $r(J)=J$. (That is, $r$ is a retraction.)

(2) Let $L=\left(L_{1}, \ldots, L_{k}\right) \in \mathbf{L}_{(k)}$ be arbitrary. If $\tau$ is a permutation of $(1, \ldots, k)$, write $\tau L=\left(L_{\tau(1)}, \ldots, L_{\tau(k)}\right)$. Then $r(\tau L)=\tau r(L)$. 
A similar result holds for $\mathbf{L}_{\{k\}}$ and $\mathbf{J}_{\{k\}}$.

Proof. $r$ is defined as follows. Let $L=\left(L_{1}, \ldots, L_{k}\right) \in \mathbf{L}_{(k)}$. Let $z_{i}$ be a unit vector in $L_{i}(i=1, \ldots, k)$. Let $Z^{2 k \times k}$ be the matrix whose $i^{t h}$ row is $z_{i}$ and whose $(k+i)^{t h}$ row is $-z_{i}(i=1, \ldots, k)$. So $Z$ has rank $k$ and the columns of $Z$ each have mean 0. By the singular value decomposition (10, Section 2.5.3]), we may write $Z=N \Lambda Q$, where $N^{2 k \times k}$ has orthonormal columns, $\Lambda^{k \times k}$ is a diagonal matrix with diagonal entries $\lambda_{1} \geq \cdots \geq \lambda_{k}>0$, and $Q^{k \times k}$ is orthogonal. Let $N_{1}^{k \times k}$ be the matrix consisting of the first $k$ rows of $N$. Then $\left(N_{1} Q\right)^{k \times k}$ is orthogonal. Define $r(L) \in \mathbf{J}_{(k)}$ to be the $k$-tuple of lines spanned by the individual rows of $N_{1} Q$. For proof that $r$ is well-defined and has the properties claimed, see [7, Proposition $2.1]$.

Finally, note that all the spaces $\mathbf{L}_{(k)}, \mathbf{J}_{(k)}, \mathbf{L}_{\{k\}}$, and $\mathbf{J}_{\{k\}}$ are homeomorphic to differentiable manifolds.

2.2. $\mathbf{L}$ and $\mathbf{J}$ bundles. Let $\zeta$ be a $k$-plane bundle over a locally compact Hausdorff space $B([17])$. Let $E(\zeta)$ and $p$ be the total space and projection, respectively (resp.), of $\zeta$. First, we define the "(ordered) $\mathbf{L}$ bundle, $\mathbf{L}_{(k)}(\zeta)$, over $B$ subordinate to $\zeta$ ". The total space $E\left[\mathbf{L}_{(k)}(\zeta)\right]$ consists of all pairs $X=\left(b,\left(L_{1}, \ldots, L_{k}\right)\right)$ s.t. $L_{1}, \ldots, L_{k}$ are linearly independent lines in $p^{-1}(b)$, the fiber of $\zeta$ over $b$. The bundle projection of $\mathbf{L}_{(k)}(\zeta)$ is $\hat{p}: X \mapsto b$.

Next, we topologize $E\left[\mathbf{L}_{(k)}(\zeta)\right]$. Let

$$
\begin{aligned}
E^{\prime}=\left\{\left(b,\left(v_{1}, \ldots, v_{k}\right)\right)\right. & \in B \times E(\zeta)^{k}: \\
& \left.v_{1}, \ldots, v_{k} \text { are linearly independent vectors in } p^{-1}(b)\right\} .
\end{aligned}
$$

Let $\sigma: E^{\prime} \rightarrow E\left[\mathbf{L}_{(k)}(\zeta)\right]$ be the projection $\sigma\left[\left(b,\left(v_{1}, \ldots, v_{k}\right)\right)\right]=\left(b,\left(\left\langle v_{1}\right\rangle, \ldots,\left\langle v_{k}\right\rangle\right)\right)$. Here, "〈·" indicates linear span. Give $E\left[\mathbf{L}_{(k)}(\zeta)\right]$ the quotient topology induced by $\sigma$. With this topology, $\mathbf{L}_{(k)}(\zeta)=\left(E\left[\mathbf{L}_{(k)}(\zeta)\right], B, \mathbf{L}_{(k)}, \hat{p}\right)$ is a fiber bundle $([20$ p. 90]).

As for unordered collections of lines, define $E\left[\mathbf{L}_{\{k\}}(\zeta)\right]$ to be the collection

$$
\left\{\left(b,\left\{L_{1}, \ldots, L_{k}\right\}\right): b \in B \text { and } L_{1}, \ldots, L_{k}\right. \text { are }
$$

linearly independent lines in $\left.p^{-1}(b)\right\}$.

Define $\tilde{p}: E\left[\mathbf{L}_{\{k\}}(\zeta)\right] \rightarrow B$ by $\left.\tilde{p}\left(b,\left\{L_{1}, \ldots, L_{k}\right)\right\}\right)=b$. Define $\rho: E\left[\mathbf{L}_{(k)}(\zeta)\right] \rightarrow$ $E\left[\mathbf{L}_{\{k\}}(\zeta)\right]$ by

$$
\rho:\left(b,\left(L_{1}, \ldots, L_{k}\right)\right) \mapsto\left(b,\left\{L_{1}, \ldots, L_{k}\right\}\right) .
$$

Put on $E\left[\mathbf{L}_{\{k\}}(\zeta)\right]$ the quotient topology generated by $\rho$. Thus, $\rho$ is a covering projection ([16, pp. 145-146]). The "un ordered bundle, $\mathbf{L}_{\{k\}}(\zeta)$, over $B$ subordinate to $\zeta$ " is the fiber bundle $\left(E\left[\mathbf{L}_{\{k\}}(\zeta)\right], B, \mathbf{L}_{\{k\}}, \tilde{p}\right)$.

Now suppose $\zeta$ is a Euclidean vector bundle $\left(\left[17\right.\right.$, p. 21]). Let $\mathbf{J}_{(k)}(\zeta)$ be the bundle with total space

$$
E\left[\mathbf{J}_{(k)}(\zeta)\right]=\left\{(b, L) \in E\left[\mathbf{L}_{(k)}(\zeta)\right]: \text { the } k \text { lines in } L \text { are orthogonal }\right\} .
$$


The fiber is $\mathbf{J}_{(k)}$ (subsection 2.1) and the projection is the restriction of the map $\hat{p}$ to $E\left[\mathbf{J}_{(k)}(\zeta)\right]$. We will continue to denote it by $\hat{p}$. Define the unordered ("jack") bundle, $\mathbf{J}_{\{k\}}(\zeta)$, with fiber $\mathbf{J}_{\{k\}}$, similarly. Denote its projection by $\tilde{p}$.

\section{ORDERED FACTOR ANALYSES}

3.1. Factor analysis on vector bundles. Let $k>1$ be an integer, and let $\eta$ be a $k$-plane bundle over a space $B$. Then an "ordered factor analysis (with $k$ factors) on $\eta$ " (or "over $B$ ") is a function, $\phi$, not necessarily continuous, that maps $B$ into $E\left[\mathbf{L}_{(k)}(\eta)\right]$ s.t. $\hat{p} \circ \phi$ is the identity on $B$. For example, if $F_{(k)}: \mathcal{Y}^{\prime} \rightarrow \mathbf{L}_{(k)}$ is as in section 1, the corresponding factor space function, $\Phi$, takes $Y \in \mathcal{Y}^{\prime}$ to the direct sum of $F_{(k)}(Y)$. $\Phi$ determines a $k$-plane bundle, $\eta$, over $\mathcal{X}$, where $\mathcal{X} \subset \overline{\mathcal{Y}^{\prime}}$ is defined as in section 1. (The fiber over $Y \in \mathcal{X}$ is $\Phi(Y)$.) $\phi: Y \mapsto\left(Y, F_{k}(Y)\right) \in E\left[\mathbf{L}_{(k)}(\eta)\right]$ is an ordered factor analysis on $\eta$. An "unordered factor analysis (with $k$ factors) on $\eta$ " (or "over $B$ ") is defined similarly. The question we are interested in here is whether a continuous ordered factor analysis on $\eta$ might exist. (A continuous factor analysis is just a section of $\mathbf{L}_{(k)}(\eta)$.) Note that if a continuous factor analysis, $\phi$, over $B$ exists for some value of $k$, then one can construct continuous factor analyses over $B$ for larger values of $k$ merely by augmenting $\phi$ by fixed lines independent of the lines in the range of $\phi$. (The corresponding vector bundle is the Whitney sum ([17, p. 27]) of the original bundle with a trivial bundle ([17, p. 14]) over B. See subsection 3.3 for an example.) By looking at a small Grassmann manifold, we will see that if $\eta$ is sufficiently rich, no continuous factor analyses on $\eta$ exist.

3.2. The 2-skeleton of $G(2,4)$. Let $G\left(m_{1}, m_{2}\right)$ be the Grassmann manifold consisting of all $m_{1}$-planes (through the origin) in $\mathbb{R}^{m_{2}}\left(0<m_{1}<m_{2} ; 3\right.$. Example 2.6, pp. 63-64]) and let $G \equiv G(2,4)$. The 2 -skeleton, $G^{2}$, of a CW-complex structure for $G$ contains two 2-cells $([17, \S 6])$ whose closures are

$$
\begin{aligned}
& G_{1}^{2}=\{\mathbf{x} \in G(2,4): \mathbf{x} \text { is perpendicular to }(0,0,0,1)\}, \text { and } \\
& G_{2}^{2}=\{\mathbf{x} \in G(2,4):(1,0,0,0) \in \mathbf{x}\} .
\end{aligned}
$$

$G_{1}^{2}$ and $G_{2}^{2}$ are each homeomorphic to the projective plane, $P^{2}$.

3.3. Ordered factor analysis over $G^{2}$. If $0<m_{1}<m_{2}$, let $\gamma^{m_{1}}\left(\mathbb{R}^{m_{2}}\right)$ denote the "canonical" $m_{1}$-plane bundle over $G\left(m_{1}, m_{2}\right)$ ([17, p. 59]). That is, if $\mathbf{x} \in$ $G\left(m_{1}, m_{2}\right)$, then the fiber of $\gamma^{m_{1}}\left(\mathbb{R}^{m_{2}}\right)$ over $\mathbf{x}$ is just $\mathbf{x}$. Let $\gamma_{2}^{2}$ be the restriction to $G^{2}$ of $\gamma^{2} \equiv \gamma^{2}\left(\mathbb{R}^{4}\right)$. If $k=2$, let $\zeta=\zeta_{2}=\gamma_{2}^{2}$. If $k>2$, let $\epsilon_{G^{2}}^{k-2}$ be the trivial $(k-2)$-plane bundle over $G^{2}$ and let $\zeta=\zeta_{k}$ be the Whitney sum $\epsilon_{G^{2}}^{k-2} \oplus \gamma_{2}^{2}$. It is isomorphic to the bundle whose total space is

$$
E\left(\zeta_{k}\right)=\left\{(\mathbf{x}, w) \in G^{2} \times \mathbb{R}^{k+2}: w=(u, v), \text { where } u \in \mathbb{R}^{k-2} \text { and } v \in \mathbf{x} \in G^{2}\right\}
$$

and whose projection is $p:(\mathbf{x}, w) \mapsto \mathbf{x} \in G^{2}$. We will prove the following.

3.1. Theorem. There does not exist a continuous ordered factor analysis with $k$ factors on $\zeta_{k}$.

3.2. Corollary. Let $\phi$ be an ordered factor analysis on a $k$-plane bundle, $\eta$, over a Hausdorff space, B. Let $f$ be a continuous map of $G^{2}$ into $B$, and suppose that the bundle over $G^{2}$ induced by $f$ is isomorphic to $\zeta_{k}$. Then $\phi$ cannot be continuous. Specifically, $\phi$ is not continuous on the restriction of $\eta$ to the image of $G^{2}$ under $f$. 
Since $\zeta_{k}$ is a rather small bundle, the hypotheses of the corollary should often be satisfied in practice. See section 7 for examples.

Proof of Theorem [3.1. We use the theory of characteristic cohomology classes of vector bundles (17]). Suppose that there does exist a continuous ordered factor analysis, $\phi$, with $k$ factors on $\zeta$. If $\mathbf{x} \in G^{2}$, we can write $\phi(\mathbf{x})=\left(\mathbf{x},\left(L_{1}(\mathbf{x}), \ldots\right.\right.$, $\left.\left.L_{k}(\mathbf{x})\right)\right)$, where $L_{1}(\mathbf{x}), \ldots, L_{k}(\mathbf{x})$ are linearly independent lines in the fiber $p^{-1}(\mathbf{x})$ and $L_{1}, \ldots, L_{k}$ are continuous. $L_{i}(\mathbf{x})$ is clearly the fiber of a subbundle, $\zeta^{i}$, of $\zeta(i=1, \ldots, k)$. The bundle $\zeta$ is isomorphic to the Whitney sum $\zeta^{1} \oplus \cdots \oplus \zeta^{k}$ (17, Lemma 3.2 , p. 28]). We show that this is impossible, thereby proving the theorem.

If $\eta$ is a vector bundle over a space $B$, let $w_{j}(\eta)\left[\bar{w}_{j}(\eta)\right] \in H^{j}(B ; \mathbb{Z} / 2)(\mathbb{Z}=$ integers; $\mathbb{Z} / 2=\{0,1\})$ denote the [dual] Stiefel-Whitney classes of $\eta$ in dimension $j=0,1,2, \ldots([17, \S 4])$. By [22, p. 69] or [17, Problem 7-B, p. 87], the cohomology algebra, $H^{*}(G ; \mathbb{Z} / 2)$, of $G$ is generated by $w_{j}\left(\gamma^{2}\right)(j=1,2)$ and the dual classes $\bar{w}_{j}\left(\gamma^{2}\right)(j=1,2)$, subject only to the four defining relations summarized by

$$
\left[1+w_{1}\left(\gamma^{2}\right)+w_{2}\left(\gamma^{2}\right)\right]\left[1+\bar{w}_{1}\left(\gamma^{2}\right)+\bar{w}_{2}\left(\gamma^{2}\right)\right]=1 .
$$

It follows that

$$
\begin{aligned}
& H^{1}(G ; \mathbb{Z} / 2) \text { is generated by } \bar{w}_{1}\left(\gamma^{2}\right)=w_{1}\left(\gamma^{2}\right) \neq 0, \\
& w_{2}\left(\gamma^{2}\right) \neq 0 \text {, and } w_{2}\left(\gamma^{2}\right) \neq w_{1}\left(\gamma^{2}\right)^{2} .
\end{aligned}
$$

Clearly, inclusion $i: G^{2} \rightarrow G$ is covered by a bundle map from $\gamma_{2}^{2}$ to $\gamma^{2}$. Therefore, $w_{j}\left(\gamma_{2}^{2}\right)=i^{*}\left[w_{j}\left(\gamma^{2}\right)\right]$. Hence, since $G^{2}$ is the 2-skeleton of $G$ we see that (3.3) holds with $\gamma^{2}$ replaced by $\gamma_{2}^{2}$.

By the Whitney product theorem ([17, pp. 37-38]), the Stiefel-Whitney classes of $\zeta$ are the same as those of $\gamma_{2}^{2}$. It thus follows from (3.3) that

$$
\begin{gathered}
H^{1}\left(G^{2} ; \mathbb{Z} / 2\right) \text { is generated by } w_{1}(\zeta) \neq 0, \\
w_{2}(\zeta) \neq 0 \text { and } w_{2}(\zeta) \neq w_{1}(\zeta)^{2} .
\end{gathered}
$$

Suppose $\zeta=\zeta^{1} \oplus \cdots \oplus \zeta^{k}$. Then, by the Whitney product theorem again,

$$
w_{1}(\zeta)=w_{1}\left(\zeta^{1}\right)+\cdots+w_{1}\left(\zeta^{k}\right) \text { and } w_{2}(\zeta)=\sum_{i<j} w_{1}\left(\zeta^{i}\right) w_{1}\left(\zeta^{j}\right) .
$$

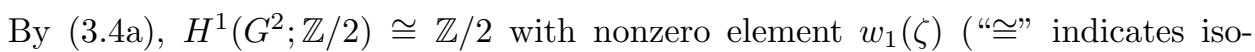
morphism). Hence, either $w_{1}\left(\zeta^{j}\right)=0$ or $w_{1}\left(\zeta^{j}\right)=w_{1}(\zeta)(j=1, \ldots, k)$. Thus, $w_{2}(\zeta)=0$ or $w_{2}(\zeta)=w_{1}(\zeta)^{2}$. But this contradicts (3.4b).

\section{The fundamental group of $\mathbf{J}_{\{3\}}$}

We turn now to unordered factor analysis. We will show that for $k=2$ or 3 a result like Corollary 3.2 holds for unordered factor analysis. This is done is section 6. This section and the next lay the groundwork.

To compute the fundamental group of $\mathbf{J}_{\{3\}}$, we use some theory of rotation in $\mathbb{R}^{3}$. See [1] or [7] for details. Define an action by the unitary group $\mathrm{SU}(2)$ on $\mathbf{J}_{\{3\}}$ as follows. Let $\mathbb{C}$ be the complex plane and $S^{2}=\left\{x \in \mathbb{R}^{3}:|x|=1\right\}$ the 2-sphere. Let $s: \mathbb{C}^{*} \equiv \mathbb{C} \cup\{\infty\} \rightarrow S^{2}$ be the stereographic projection that takes $u+i v \in \mathbb{C}$ to the point (other than $(0,0,-1)$ itself) where the line joining $(u, v, 0) \in \mathbb{R}^{3}$ and 
$(0,0,-1) \in S^{2}$ intersects $S^{2}$. Let $s(\infty)=(0,0,-1)$. If $A=\left(\begin{array}{cc}a & b \\ -\bar{b} & \frac{b}{a}\end{array}\right) \in \mathrm{SU}(2)$, let $f_{A}: \mathbb{C}^{*} \rightarrow \mathbb{C}^{*}$ be the linear fractional transformation determined by $A$, i.e., $f_{A}(w)=(a w+b) /(-\bar{b} w+\bar{a})$. Let $R_{A}: \mathbb{R}^{3} \rightarrow \mathbb{R}^{3}$ be the linear map corresponding to the rotation $s \circ f_{A} \circ s^{-1}$ of $S^{2}$. If $J \in \mathbf{J}_{\{3\}}$, write $J=\left\{L_{1}, L_{2}, L_{3}\right\}$, where $L_{1}, L_{2}, L_{3}$ are orthogonal lines in $\mathbb{R}^{3}$. Define $R_{A}\left(L_{i}\right)$ pointwise, and let

$$
\theta(A, J)=\left\{R_{A}\left(L_{1}\right), R_{A}\left(L_{2}\right), R_{A}\left(L_{3}\right)\right\} \in \mathbf{J}_{\{3\}} .
$$

Then $\theta$ defines a $C^{\infty}$ group action on $\mathbf{J}_{\{3\}}$ ([3] Section 7 , Chapter III]) and is transitive.

Let $J^{\{0\}} \in \mathbf{J}_{\{3\}}$ be the set of the three standard coordinate axes in $\mathbb{R}^{3}$. Let $\mathbf{O}$ be the isotropy group of $J^{\{0\}}$ in $\mathrm{SU}(2)$. $\mathbf{O}$ is the "binary octahedral group" ([4, pp. 538-539], [21, p. 20]). $\mathbf{O}$ consists of 48 matrices and is generated by the matrices

$$
A_{1}=\frac{1}{\sqrt{2}}\left(\begin{array}{cc}
1 & -i \\
-i & 1
\end{array}\right), A_{2}=\frac{1}{\sqrt{2}}\left(\begin{array}{cc}
1 & 1 \\
-1 & 1
\end{array}\right) \text {, and } A_{3}=\frac{1}{\sqrt{2}}\left(\begin{array}{cc}
1+i & 0 \\
0 & 1-i
\end{array}\right)
$$

( $A_{i}$ corresponds to a $90^{\circ}$ rotation about the $i^{\text {th }}$ coordinate axis). Actually, only two of these are needed to generate the group. For example, $A_{3}=A_{1}^{-1} A_{2}^{-1} A_{1}$. Note that

$$
A_{i}^{4}=\left(\begin{array}{cc}
-1 & 0 \\
0 & -1
\end{array}\right) \text {, so the period of } A_{i} \text { is } 8(i=1,2 \text {, or } 3)
$$

The spaces $\mathrm{SU}(2) / \mathbf{O}$ and $\mathbf{J}_{\{3\}}$ are homeomorphic ([3, Theorem 9.3, p. 166]). Since $\mathbf{O}$ is finite and $\mathrm{SU}(2)$ is simply connected, $\pi_{1}\left(\mathbf{J}_{\{3\}}\right) \cong \mathbf{O}(20$, Corollary $8, \mathrm{p}$. $88])$.

\section{Fundamental group of the total space of a Jack Bundle}

5.1. Boundary map in exact homotopy sequence of fibration. Define $G_{1}^{2}$ as in subsection 3.2. Let $\xi$ be the restriction of the 3-plane bundle $\zeta_{3}$ (subsection 3.3) to $G_{1}^{2}$. Our goal in this section is to compute the fundamental group of $E=E\left[\mathbf{J}_{\{3\}}(\xi)\right]$, the total space of $\mathbf{J}_{\{3\}}(\xi)$ (subsection [2.2).

Specify base points in the spaces. Let the base point of $G_{1}^{2}$ be

$$
\mathbf{x}_{0}=\langle(0,1,0,0),(0,0,1,0)\rangle \in G_{1}^{2} .
$$

(Recall that "〈·〉" = "linear span.") Identify $\mathbf{J}_{\{3\}}$ (subsection2.1) with $\tilde{p}^{-1}\left(\mathbf{x}_{0}\right) \in E$ (section 2.2) via the linear map, $\lambda$, from $\mathbb{R}^{3}$ to $\mathbb{R}^{5}$ determined by

$$
(1,0,0) \mapsto(1,0,0,0,0),(0,1,0) \mapsto(0,0,1,0,0),(0,0,1) \mapsto(0,0,0,1,0) .
$$

Thus, $\left\{L_{1}, L_{2}, L_{3}\right\} \in \mathbf{J}_{\{3\}}$ is identified with $\left(\mathbf{x}_{0},\left\{\lambda\left(L_{1}\right), \lambda\left(L_{2}\right), \lambda\left(L_{3}\right)\right\}\right)$. ( $\lambda$ is applied pointwise.) Let the base point of $\mathbf{J}_{\{3\}}$ and $E$ be

$$
J^{\{0\}}=\left(\mathbf{x}_{0},\{\langle(1,0,0,0,0)\rangle,\langle(0,0,1,0,0)\rangle,\langle(0,0,0,1,0)\rangle\}\right) .
$$

By [20. Corollary 14, p. 96], the projection $\tilde{p}: E \rightarrow G_{1}^{2}$ is a fibration. Therefore, by [20, Theorem 10, p. 377], we have an exact sequence

$$
\pi_{2}\left(G_{1}^{2}\right) \stackrel{\bar{\partial}}{\rightarrow} \pi_{1}\left(\mathbf{J}_{\{3\}}\right) \stackrel{i_{\#}}{\rightarrow} \pi_{1}(E) \stackrel{\tilde{p}_{\#}}{\rightarrow} \pi_{1}\left(G_{1}^{2}\right) \stackrel{\bar{\partial}}{\rightarrow} \pi_{0}\left(\mathbf{J}_{\{3\}}\right)
$$

of homotopy groups, where $i: \mathbf{J}_{\{3\}}=\tilde{p}^{-1}\left(\mathbf{x}_{0}\right) \rightarrow E$ is inclusion. In this subsection we calculate $\bar{\partial}: \pi_{2}\left(G_{1}^{2}\right) \rightarrow \pi_{1}\left(\mathbf{J}_{\{3\}}\right)$.

First, we describe $\pi_{2}\left(G_{1}^{2}\right) \cong \pi_{2}\left(P^{2}\right)$ ([11, p. 36]). Let the base point for $S^{2}$ be $x_{0}=(1,0,0)$. Let $I$ be the closed interval $[0,1]$ and let $\dot{I}^{j}$ be the boundary of $I^{j}$, 
both with base point $z_{0}=(0, \ldots, 0)(j=1,2, \ldots)$. Define a map $g_{1}:\left(I^{2}, \dot{I}^{2}\right) \rightarrow$ $\left(S^{2}, x_{0}\right)$ as follows:

$$
g_{1}(s, t)=\left(\cos ^{2} \pi s+\sin ^{2} \pi s \cos 2 \pi t, \sin 2 \pi s \sin ^{2} \pi t, \sin \pi s \sin 2 \pi t\right), \quad s, t \in I .
$$

Geometrically, $g_{1}$ is defined as follows. Consider the circle, $C(s)$, on $S^{2}$ passing through $x_{0}$ and $a(s)=(\cos 2 \pi s, \sin 2 \pi s, 0)$ with center at $w(s)=\frac{1}{2}\left(x_{0}+a(s)\right)$. As $t$ varies from 0 to $1, g_{1}(s, t)$ goes around $C(s)$ once, starting and stopping at $x_{0}$. Let $[s, t]$ be the point of the suspension, $S I$, corresponding to $(s, t) \in I^{2}$ ([20, p. $41])$, and let $\mathbf{x}([s, t]) \in G_{1}^{2}$ be the 2-plane in $\mathbb{R}^{4}$ perpendicular to both $\left(g_{1}(s, t), 0\right)$ and $(0,0,0,1)$. The map x $: S(I, \dot{I}) \rightarrow\left(G_{1}^{2},\left\{\mathbf{x}_{0}\right\}\right)([20$, pp. 368, 372]) represents a generator, $\beta$, of $\pi_{2}\left(G_{1}^{2}\right) \cong \mathbb{Z}$.

To compute $\bar{\partial}$ it suffices to determine $\bar{\partial}(\beta)$. The first step is to find an element of $\pi_{2}\left(E, \mathbf{J}_{\{3\}}\right)$ that $\tilde{p}_{\#}$ takes to $\beta$. We will construct a representative, $X_{2}: S(I, \dot{I}) \rightarrow$ $\left(E, \mathbf{J}_{\{3\}}\right)$, of this element. For $s, t \in I$, the partial derivative, $D_{2} g_{1}(s, t) \in \mathbb{R}^{3}$, of $g_{1}(s, t)$ with respect to $t$ is perpendicular to $g_{1}(s, t)$. Let

$$
u(s, t)=\left|D_{2} g_{1}(s, t)\right|^{-1} D_{2} g_{1}(s, t)=(-\sin \pi s \sin 2 \pi t, \cos \pi s \sin 2 \pi t, \cos 2 \pi t),
$$

where the right-most member of this equation is used as the definition of $u(s, t)$ even when $D_{2} g_{1}(s, t)=0$. Let

$v(s, t)=g_{1}(s, t) \times u(s, t)$ and $x(s, t)=\cos 2 \pi t u(s, t)+\sin 2 \pi t v(s, t) \in \mathbb{R}^{3}, \quad s, t \in I$,

where " $\times$ " indicates vector cross product. Of course, $x(s, t)$ is a unit vector in $\mathbb{R}^{3}$ perpendicular to $g_{1}(s, t)$. Let

$$
y(s, t)=g_{1}(s, t) \times x(s, t), \quad s, t \in I .
$$

One easily sees that $x$ and $y$ induce maps on $S(I, \dot{I})$. Finally, let

$X_{2}([s, t])=(\mathbf{x}([s, t]),\{\langle(1,0,0,0,0)\rangle,\langle(0, x(s, t), 0)\rangle,\langle(0, y(s, t), 0)\rangle\}) \in E, \quad s, t \in I$.

Trivially, $\tilde{p} \circ X_{2}=\mathbf{x}$. So $\tilde{p}_{\#}\left(\left[X_{2}\right]\right)=[\mathbf{x}]=\beta$, where $\left[X_{2}\right] \in \pi_{2}(E)\left([\mathbf{x}] \in \pi_{2}\left(G_{1}^{2}\right)\right)$ are the homotopy classes containing $X_{2}$ (resp., $\mathbf{x}$ ).

To finish computing $\bar{\partial}(\beta)$, we need to compute the homotopy class $\partial\left(\left[X_{2}\right]\right) \in$ $\pi_{1}\left(\mathbf{J}_{\{3\}}\right)$, where $\partial\left(\left[X_{2}\right]\right)$ is just the homotopy class of the restriction, $\left.X_{2}\right|_{S(\dot{I})}$, of $X_{2}$ to $S(\dot{I})$ :

$$
\begin{aligned}
& X_{2}([1, t]) \\
= & \left(\mathbf{x}_{0},\{\langle(1,0,0,0,0)\rangle,\langle(0,0,-\sin 4 \pi t, \cos 4 \pi t, 0)\rangle,\langle(0,0, \cos 4 \pi t, \sin 4 \pi t, 0)\rangle\}\right),
\end{aligned}
$$

$t \in I$. Thus, $\left.X_{2}\right|_{S(I)}$ amounts to rotating the jack $J^{\{0\}}$ counterclockwise about $(1,0,0,0,0)$, (and $(0,1,0,0,0)$, and $(0,0,0,0,1))$ through an angle of $4 \pi$.

Now, from section 4, under the identification (5.2), counterclockwise rotation of $J^{\{0\}}$ about $(1,0,0,0,0)$ through $\pi / 2$ radians is represented by the element $A_{1}$, defined in (4.2). Thus, $\bar{\partial}(\beta)$ is represented by $A_{1}^{8}$. But, by (4.3) $A_{1}^{8}$ is just the identity matrix. Thus, $\bar{\partial}$ is trivial. 
5.2. Fundamental group of $E\left[\mathbf{J}_{\{3\}}(\xi)\right]$. From the last section, especially (5.4), we arrive at the exact sequence

$$
1 \rightarrow \pi_{1}\left(\mathbf{J}_{\{3\}}\right) \stackrel{i_{\#}}{\rightarrow} \pi_{1}(E) \stackrel{\tilde{p}_{\#}}{\rightarrow} \pi_{1}\left(G_{1}^{2}\right) \rightarrow 1,
$$

where $E=E\left[\mathbf{J}_{\{3\}}(\xi)\right]$, on the left "1" is the identity element of $\pi_{2}\left(G_{1}^{2}\right)$, and on the right " 1 " is the single path component of $\mathbf{J}_{\{3\}}$.

Using the theorem of Seifert and Van Kampen (16, Theorem 2.1, p. 114]), we find that

$$
\pi_{1}\left(G^{2}\right) \cong \mathbb{Z} / 2 \text { and } j_{1 \#}: \pi_{1}\left(G_{1}^{2}\right) \rightarrow \pi_{1}\left(G^{2}\right) \text { is an isomorphism, }
$$

where $j_{1}: G_{1}^{2} \rightarrow G^{2}$ is inclusion. For $s \in I$, let $\mathbf{y}(s) \in G_{1}^{2}$ be the 2-plane in $\mathbb{R}^{4}$ perpendicular to $(0,0,0,1)$ and $(\cos \pi s, \sin \pi s, 0,0)$. $\mathbf{y}$ represents the generator of $\pi_{1}\left(G_{1}^{2}\right) \cong \pi_{1}\left(P^{2}\right)$ (16, Example 5.2, pp. 130-131]). For $s \in I$, let

$$
X_{1}(s)=(\mathbf{y}(s),\{\langle(1,0,0,0,0)\rangle,\langle(0,-\sin \pi s, \cos \pi s, 0,0)\rangle,\langle(0,0,0,1,0)\rangle\}) \in E .
$$

Then $X_{1}:(I, \dot{I}) \rightarrow\left(E,\left\{J^{\{0\}}\right\}\right)$ represents an element, $\alpha$, of $\pi_{1}(E)$ and $\tilde{p} \circ X_{1}=\mathbf{y}$. Thus,

$$
\tilde{p}_{\#}(\alpha) \text { is the generator of } \pi_{1}\left(G_{1}^{2}\right) .
$$

Let $W=i_{\#}\left(\pi_{1}\left(\mathbf{J}_{\{3\}}\right)\right)$. By (5.6) we may identify $W$ with $\mathbf{O}$ as defined in section 4. Sequence (5.6) also implies

$$
\text { every element of } \pi_{1}(E) \text { can be written in the form } w \alpha^{l} \text {, }
$$

where $w \in W$ and $l=0,1$. By explicit construction of homotopies, we find (see (4.2)) that

$$
\alpha^{2}=A_{1}^{4}, \quad \alpha^{-1}=A_{1}^{4} \alpha, \quad A_{1} \alpha=\alpha A_{1}^{-1}, \quad A_{2} \alpha=\alpha A_{2}, \quad \text { and } A_{3} \alpha=\alpha A_{3}^{-1} .
$$

\section{LifTING OF UNORDERED FACTOR ANALYSES WHEN $k=3$}

Here we will show that there can be no continuous unordered factor analysis on $\zeta=\zeta_{3}$ (subsection [3.3). The strategy of proof is as follows. We will show that if there were a continuous unordered factor analysis on $\zeta$, then there would be a continuous unordered jack field on $G^{2}$, i.e., a continuous map $\phi: G^{2} \rightarrow E\left[\mathbf{J}_{\{3\}}(\zeta)\right]$ with the property that $\tilde{p}[\phi(\mathbf{x})]=\mathbf{x}, \mathbf{x} \in G^{2} .\left(\tilde{p}: E\left[\mathbf{J}_{\{3\}}(\zeta)\right] \rightarrow G^{2}\right.$ is the bundle projection.) We will show that if there were such a jack field, then it could be lifted to $E\left[\mathbf{J}_{(3)}(\zeta)\right]$, contradicting the fact that there is no continuous ordered factor analysis on $\zeta$ (Theorem 3.1). (To say that $\phi$ can be "lifted" to $E\left[\mathbf{J}_{(3)}(\zeta)\right]$ means that there exists a continuous ordered factor analysis $\hat{\phi}: G^{2} \rightarrow E\left[\mathbf{J}_{(3)}(\zeta)\right]$ s.t. $\rho \circ \hat{\phi}=\phi$, where $\rho: E\left[\mathbf{L}_{(3)}(\zeta)\right] \rightarrow E\left[\mathbf{L}_{\{3\}}(\zeta)\right]$ is the covering projection defined by (2.1).)

Let $\phi: G^{2} \rightarrow E\left[\mathbf{L}_{\{3\}}(\zeta)\right]$ be a continuous (unordered) factor analysis on $\zeta$. It follows from subsection 2.1 that there is a continuous retract $\tilde{r}: E\left[\mathbf{L}_{\{3\}}(\zeta)\right] \rightarrow$ $E\left[\mathbf{J}_{\{3\}}(\zeta)\right]$ with the property that $\tilde{p} \circ \tilde{r}=\tilde{p}$. Hence, we may assume, without loss of generality, that $\phi$ maps $G^{2}$ into $E\left[\mathbf{J}_{\{3\}}(\zeta)\right]$.

By [16, Theorem 5.1, p. 156], the jack field $\phi$ can be lifted to $E\left[\mathbf{J}_{(3)}(\zeta)\right]$ if and only if

$$
\phi_{\#}\left[\pi_{1}\left(G^{2}\right)\right] \subset \rho_{\#}\left[\pi_{1}\left(E\left[\mathbf{J}_{(3)}(\zeta)\right]\right)\right] \subset \pi_{1}\left(E\left[\mathbf{J}_{\{3\}}(\zeta)\right]\right)
$$


First, we show that it suffices to prove the result with $G_{1}^{2} \subset G^{2}$ in place of $G^{2}$. Let $\xi$ be the 3-plane bundle over $G_{1}^{2}$ defined in subsection 5.1 Let the base points of $G_{1}^{2}$ and $G^{2}$ be the plane $\mathbf{x}_{0}$ defined in (5.1). Define the base points of $E\left[\mathbf{J}_{\{3\}}(\xi)\right]$ and $E\left[\mathbf{J}_{\{3\}}(\zeta)\right]$ by (5.3). Define the base points of $E\left[\mathbf{J}_{(3)}(\xi)\right]$ and $E\left[\mathbf{J}_{(3)}(\zeta)\right]$ as in (5.3), but with " $\{\cdot\}$ " replaced by "(.)".

Let $j_{1}: G_{1}^{2} \rightarrow G^{2}, \tilde{j}_{1}: E\left[\mathbf{J}_{\{3\}}(\xi)\right] \rightarrow E\left[\mathbf{J}_{\{3\}}(\zeta)\right]$, and $\hat{j}_{1}: E\left[\mathbf{J}_{(3)}(\xi)\right] \rightarrow E\left[\mathbf{J}_{(3)}(\zeta)\right]$ be inclusions. Let $\tilde{p}_{\xi}, \rho_{\xi}$, and $\phi_{\xi}$ be the restrictions of $\tilde{p}$ to $E\left[\mathbf{J}_{\{3\}}(\xi)\right]$, $\rho$ to $E\left[\mathbf{J}_{(3)}(\xi)\right]$, and $\phi$ to $G_{1}^{2}$, resp. Then

$$
\tilde{p}_{\xi \#} \circ \phi_{\xi \#} \text { is the identity on } \pi_{1}\left(G_{1}^{2}\right),
$$

but $\phi_{\xi \#} \circ \tilde{p}_{\xi \#}$ is not the identity on $\pi_{1}\left(E\left[\mathbf{J}_{\{3\}}(\xi)\right]\right)$. Similarly for $\phi_{\#}$ and $\tilde{p}_{\#}$. With those exceptions, the following diagram commutes:

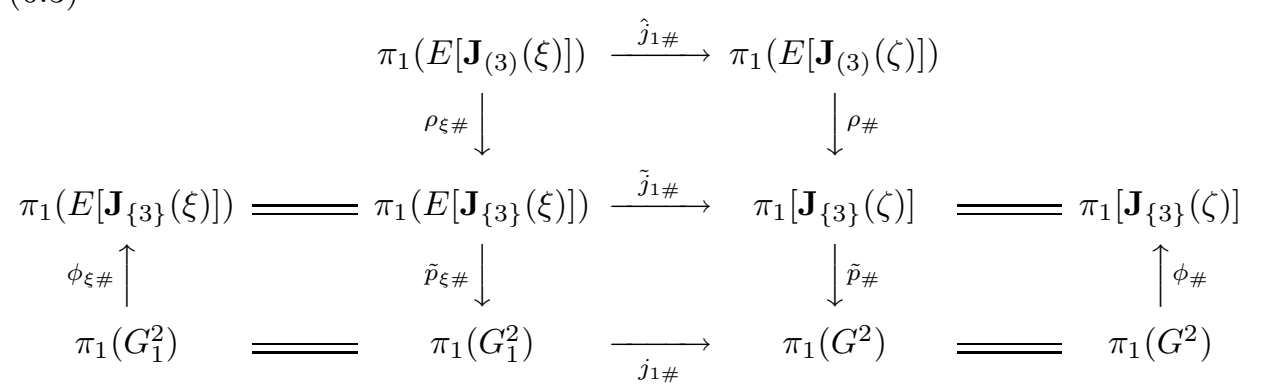

Suppose the analogue of (6.1) holds for $G_{1}^{2}$, viz.,

$$
\phi_{\xi \#}\left[\pi_{1}\left(G_{1}^{2}\right)\right] \subset \rho_{\xi \#}\left[\pi_{1}\left(E\left[\mathbf{J}_{(3)}(\xi)\right]\right)\right] \subset \pi_{1}\left(E\left[\mathbf{J}_{\{3\}}(\xi)\right]\right) .
$$

Let $a_{1}, a$ be the generators of $\pi_{1}\left(G_{1}^{2}\right), \pi_{1}\left(G^{2}\right)$, resp. (see (5.7)). By (5.7), $j_{1 \#}\left(a_{1}\right)=$ a. Thus, by 6.4,

$$
\phi_{\xi \#}\left(a_{1}\right) \in \rho_{\xi \#}\left[\pi_{1}\left(E\left[\mathbf{J}_{(3)}(\xi)\right]\right)\right] .
$$

A chase around the diagram ([6.3) shows that $\phi_{\#}(a) \in \rho_{\#}\left[\pi_{1}\left(E\left[\mathbf{J}_{(3)}(\zeta)\right]\right)\right]$. That is, 6.1 holds.

Thus, to prove that $\phi$ can be lifted, it suffices to prove (6.4). In fact, it suffices to prove (6.5). Let $b_{1}=\phi_{\xi \#}\left(a_{1}\right) \in \pi_{1}\left(E\left[\mathbf{J}_{\{3\}}(\xi)\right]\right)$. By (5.9) we can write $b_{1}=w \alpha^{l}$, where $w \in W=\pi_{1}\left(\mathbf{J}_{\{3\}}\right) \cong \mathbf{O}$ and $l=0,1$. Now, by (6.2) and (5.8), we see that $l=1$. By inspection of the 48 elements of $\mathbf{O}$ and using (5.10), we find that the only choices of $w$ satisfying

$$
(w \alpha)^{2}=b_{1}^{2}=\phi_{\xi \#}\left(a_{1}\right)^{2}=\phi_{\xi \#}\left(a_{1}^{2}\right)=1
$$

satisfy $b_{1} \in \rho_{\xi \#}\left[\pi_{1}\left(E\left[\mathbf{J}_{(3)}(\xi)\right]\right)\right]$ (see [7] for details). Therefore, (6.5) holds. Hence, no section $\phi$ is possible.

To sum up, we have the following. (The $k=3$ result implies the $k=2$ result.) I conjecture that the result holds for any $k>1$.

6.1. Theorem. Suppose $k=2$ or 3. Let $\phi$ be an unordered factor analysis on a $k$-plane bundle, $\eta$, over a Hausdorff space, B. Let $f$ be a continuous map of $G^{2}$ into $B$ and suppose the bundle over $G^{2}$ induced by $f$ is isomorphic to $\zeta_{k}$. Then $\phi$ cannot be continuous. Specifically, $\phi$ is not continuous on the restriction of $\eta$ to the image of $G^{2}$ under $f$. 


\section{EXAMPLES}

In this section we consider factor analysis methods that fit model (1.2) with $C=I_{k}$. A desirable quality for a factor analysis method is that it recognize perfect fits (section 1), provided they are identifiable. [2, (4.5)] finds the following necessary condition on $k$ and $p$ so that, if (1.2) holds exactly with $C=I_{k}$, the column space of $K$ (i.e., the factor space) and $D$ are identifiable from $S$ (actually, it is the reverse of [2, (4.5)] that is needed):

$$
k \leq p-\frac{\sqrt{8 p+1}-1}{2}<p .
$$

(See also [15, Section 2.3].) From another point of view, if (7.1) holds strictly, then (1.2) provides a representation for $S$ containing fewer independent parameters than $S$ does. In Example 7.1 we adopt

$$
1<k<p-2 .
$$

In Example 7.2 we assume

$$
p \geq 2 k+9 \text { and } n \geq p+k+8 .
$$

(1.1) remains in force.) (7.2) and (7.3) are often compatible with (7.1). Indeed, (1.1) and (7.1) imply (7.2). (7.3) implies (7.1).

Let $F$ be a factor analysis method in the traditional sense, viz., a function defined on a subset of $\mathcal{Y}$ and taking values in the space of $(p \times k)$-dimensional matrices. As described in section 1, $F$ can be interpreted as a function whose values are $k$ tuples or collections of lines. Let $\mathcal{Y}^{\prime}=\{Y \in \mathcal{Y}: F(Y)$ is uniquely defined $\}$ (where "unique" means unique up to the signs of the columns of $F(Y)$ ), and let $\Phi^{\prime}(Y)$ be the factor space of $F(Y), Y \in \mathcal{Y}^{\prime}$ (see section 11). Let $\mathcal{X}$ be the collection of all $Y \in \overline{\mathcal{Y}^{\prime}}$ at which $\lim _{Y^{\prime} \rightarrow Y, Y \in \mathcal{Y}^{\prime}} \Phi^{\prime}(Y)$ exists. Let $\Phi(Y)=\lim _{Y^{\prime} \rightarrow Y, Y \in \mathcal{Y}^{\prime}} \Phi^{\prime}(Y)$, $Y \in \mathcal{X}$.

Recall that $\gamma^{k}\left(\mathbb{R}^{p}\right)$ is the canonical $k$-plane bundle over the Grassmann manifold $G(k, p) . \Phi: \mathcal{X} \rightarrow G(k, p)$ determines a $k$-plane bundle, $\eta=\Phi^{*} \gamma^{k}\left(\mathbb{R}^{p}\right)$, over $\mathcal{X}$.

By Corollary 3.2 and Theorem 6.1, to prove the existence of a factor singularity, it suffices to find a continuous map $f: G^{2} \rightarrow \mathcal{X}$ satisfying the following:

$$
\text { The bundle, } f^{*} \eta \text {, over } G^{2} \text { induced by } f \text { is isomorphic to } \zeta_{k} \text {, }
$$

where $\zeta=\zeta_{k}$ is the bundle defined in subsection [3.3. (In the unordered case, we must assume $k=2$ or 3 , of course.) Condition (7.4) holds if the map

$$
\mathrm{g}=\Phi \circ f: G^{2} \rightarrow G(k, p)
$$

satisfies

$$
\mathbf{g}^{*} \gamma^{k}\left(\mathbb{R}^{p}\right) \cong \zeta_{k} .
$$

Assuming (7.2) or (7.3), we now construct, for two popular factor analysis methods, functions, $f$ and $\mathbf{g}$, satisfying (7.5). It will follow that any factor analysis method with the same factor space function as in the examples (obtained, for example, by rotation) will have a factor singularity (in the unordered case, at least if $1<k \leq 3)$. I conjecture that this remains true under assumptions milder than (7.2) and (7.3). See [13] for further examples, both algebraic and numerical. 
7.1. Example (Principal Components (Example 1.1, continued)). For any positive integer $j$, let $1_{j} \in \mathbb{R}^{j}$ be the column vector all of whose coordinates are 1 . The "mean vector" of $Y \in \mathcal{Y}$ is just $\frac{1}{n} 1_{n}^{T} Y \in \mathbb{R}^{p} . Y$ is "centered" by subtracting the mean vector from each row of $Y: Y_{0} \equiv Y-\frac{1}{n} 1_{n} 1_{n}^{T} Y$. The "(sample) covariance matrix" of $Y$ is the $p \times p$ matrix

$$
S=S(Y)=\frac{1}{n} Y_{0}^{T} Y_{0}=\frac{1}{n} Y^{T}\left(I_{n}-\frac{1}{n} 1_{n} 1_{n}^{T}\right) Y .
$$

Define "principal components factor analysis based on the covariance matrix" (PC) as in (1.4). Assume (7.2). We show that, for PC functions, $f$ and $\mathbf{g}$ exist satisfying (7.5). Using the notation of Example 1.1, take $\tilde{\Phi}(Y)$ to be the linear space spanned by $v_{1}, \ldots, v_{k}$ whenever it is defined, even if $\lambda_{1}, \ldots, \lambda_{k}$ are not distinct. So $\tilde{\Phi}(Y)=$ $\Phi(Y)$ for $Y \in \mathcal{Y}^{\prime}$.

Define $\mathbf{g}$ as follows. Let $\mathbf{x}$ and $\mathbf{z}$ be fixed orthogonal linear subspaces of $\mathbb{R}^{p}$, perpendicular to $1_{p}$ and having dimensions $k-2$ and 4 , resp.

Let $a: \mathbb{R}^{4} \rightarrow \mathbf{z}$ be a linear isomorphism. If $\mathbf{y} \in G^{2}$, define $a(\mathbf{y})$ pointwise and let $\mathbf{g}(\mathbf{y})=\mathbf{g}_{\mathbf{x}}(\mathbf{y})=\mathbf{x} \oplus a(\mathbf{y}) \in G(k, p)$. Clearly, (7.5b) holds.

If $k>2$, let $E^{p \times(k-2)}$ be a matrix whose columns form an orthonormal basis of $\mathbf{x}$ and let $\beta^{(k-2) \times(k-2)}$ be a fixed diagonal matrix whose diagonal entries are $1>\beta_{1}>\cdots>\beta_{k-2}>0$. (If $k=2$, ignore $E$ and $\beta$ in the following.) Let $\alpha^{2 \times 2}$ be the diagonal matrix with diagonal entries $\alpha_{1} \geq \alpha_{2} \geq 1$. If $\mathbf{y} \in G^{2}$, let $A^{p \times 2}=A(\mathbf{y})$ have orthonormal columns spanning $a(\mathbf{y})$. Let $f_{\alpha, \beta}(\mathbf{y}) \in \mathcal{Y}$ be the matrix

$$
f_{\alpha, \beta}(\mathbf{y})=\left(\begin{array}{c}
A \alpha A^{T}+E \beta E^{T} \\
0^{(n-p) \times p}
\end{array}\right) .
$$

Note that by choice of $\mathbf{x}$ and $\mathbf{z}$, the columns of $f(\mathbf{y})$ are mean centered. Hence, the covariance matrix, $S\left[f_{\alpha, \beta}(\mathbf{y})\right]$, of $f(\mathbf{y})$ is given by the following:

$$
S\left[f_{\alpha, \beta}(\mathbf{y})\right]=\frac{1}{n} f_{\alpha, \beta}(\mathbf{y})^{T} f_{\alpha, \beta}(\mathbf{y})=\frac{1}{n}\left(A \alpha^{2} A^{T}+E \beta^{2} E^{T}\right) .
$$

Thus, the eigenvalues of $S[f(\mathbf{y})]$ are 0 (multiplicity $p-k$ ) and $\alpha_{1}^{2} / n \geq \alpha_{2}^{2} / n>$ $\beta_{1}^{2} / n>\cdots>\beta_{k-2}^{2} / n>0$, and $f_{\alpha, \beta}(\mathbf{y})$ is a perfect fit (section 11). The span of the eigenvectors of $S[f(\mathbf{y})]$ corresponding to the first $k$ eigenvalues is just $\mathbf{g}(\mathbf{y})$. Thus, (7.5) holds with $\mathbf{g}=\mathbf{g}_{\mathbf{x}}$. If $\alpha_{1}>\alpha_{2} \geq 1$, then the $k$ principal components of $f_{\alpha, \beta}(\mathbf{y})$ are uniquely determined. So $f_{\alpha, \beta}\left(G^{2}\right) \subset \mathcal{Y}^{\prime}$, but $f_{\alpha, \beta}(\mathbf{y})$ depends on an arbitrary choice of columns for $A$. There may be no way to make this choice in a continuous fashion. If $\mathbf{w}$ is a vector subspace of $\mathbb{R}^{m}$ for some $m=1,2,3, \ldots$, denote by $\Pi_{\mathbb{R}^{m}, \mathbf{w}}$ the matrix of orthogonal projection from $\mathbb{R}^{m}$ onto $\mathbf{w}$, with respect to the standard basis of $\mathbb{R}^{m}$. If $\alpha_{1}=\alpha_{2}=1$, so $\alpha=I_{2}$, then $A \alpha A^{T}=A A^{T}=\Pi_{\mathbb{R}^{p}, a(\mathbf{y})}$. In particular, $A(\mathbf{y}) A(\mathbf{y})^{T}$ does not depend on which orthogonal basis of $a(\mathbf{y})$ is chosen as the columns of $A(\mathbf{y})$. Moreover, $\Pi_{\mathbb{R}^{p}, a(\mathbf{y})}$ is a continuous function of $\mathbf{y}$. Therefore, $f_{I_{2}, \beta}(\mathbf{y})$ is continuous. What is more, by taking $\alpha_{1}>\alpha \geq 1$ approaching 1 , we see that $f_{I_{2}, \beta}\left(G^{2}\right) \subset \overline{\mathcal{Y}^{\prime}}$. Since the rows of $f_{I_{2}, \beta}(\mathbf{y})$ lie exactly and uniquely on the $k$-plane $\mathbf{x} \oplus a(\mathbf{y})$, and $\tilde{\Phi}$ is continuous at data sets lying on unique $k$-planes, it follows that $\mathcal{W} \equiv f_{I_{2}, \beta}\left(G^{2}\right) \subset \mathcal{X}$, and $\tilde{\Phi}$ and $\Phi$ coincide on $\mathcal{W}$. Since the first two eigenvalues of $S\left[f_{I_{2}, \beta}(\mathbf{y})\right]$ are equal, every data set in $\mathcal{W}$ is a factor singularity (section 1).

7.2. Example (Maximum Likelihood). In maximum likelihood factor analysis one computes $K$ and $D$ in (1.2) (with $C=I_{k}$ ) by estimating them as parameters, $\Lambda$ 
and $\Psi$, resp., in a Gaussian model using maximum likelihood. By [15] Sections 4.1 and 4.2], any "maximum likelihood estimate" (MLE), $\left(\hat{\Lambda}^{p \times k}, \hat{\Psi}^{p \times p}\right)$, at $Y \in \mathcal{Y}$, provided it exists, satisfies

$$
\Lambda=\hat{\Lambda} \text { and } \Psi=\hat{\Psi} \text { minimize } l\left(\Lambda \Lambda^{T}+\Psi, S(Y)\right),
$$

where $S(Y)$ is the covariance matrix (7.6) and

$$
l\left(\Sigma^{p \times p}, S^{p \times p}\right)=\log \operatorname{det}(\Sigma)+\operatorname{trace}\left(S \Sigma^{-1}\right),
$$

subject to

$\Lambda$ has rank $k, \Psi$ is diagonal with strictly positive diagonal entries, and

$$
\Lambda^{T} \Psi^{-1} \Lambda \text { is diagonal with strictly decreasing diagonal entries. }
$$

Maximum likelihood factor analysis is defined at those data sets having an MLE that is unique (up to signs of the columns of $\Lambda$ ). Let $\mathcal{Y}^{\prime} \subset \mathcal{Y}$ be the collection of data sets having a unique MLE.

Call a pair $\left(\hat{\Lambda}^{p \times k}, \hat{\Psi}^{p \times p}\right)$ an "unconstrained MLE" (UMLE) at $Y$ if it satisfies (7.8) subject to the weaker constraint

$\Psi$ is diagonal with nonnegative entries.

(In this case $\hat{\Lambda}$ is a "UMLE of $\Lambda$ ".) There may be data sets at which there are UMLE's but no MLE's. However, employing the argument of [15, pp. 7,8], it is easy to see that

$$
\text { any MLE is a UMLE. }
$$

Let $\Phi$ be the factor space function (section 1) of maximum likelihood factor analysis. Thus, if $(\hat{\Lambda}, \hat{\Psi})$ is the MLE at $Y \in \mathcal{Y}^{\prime}$, then $\Phi(Y)$ is just the column space of $\hat{\Lambda}$. We will construct a continuous map, $f$, that satisfies the condition (7.5). Let $\epsilon>0$ and assume (7.3). Let $q=p-2 k-9$. Let $\mathbf{y} \in G^{2}$. Let the columns of $A^{4 \times 2}=$ $A(\mathbf{y})$ be an orthonormal basis for $\mathbf{y}$. Let $M^{(p+1) \times p}$ have orthonormal columns perpendicular to $1_{p+1}$. Let $N^{(k-1) \times(k-2)}$ have orthonormal columns orthogonal to $1_{k-1}$. Let $\alpha$ be a $2 \times 2$ diagonal matrix, $\beta$ a fixed $(k-2) \times(k-2)$ diagonal matrix with diagonal entries $1 / \sqrt{2}>\beta_{1}>\ldots>\beta_{k-2}>0$, and $b=\left(\sum_{i=1}^{k-2} \beta_{i}^{-2}\right)^{-1 / 2}$. If $k>2$, let $w=b N \beta^{-1} 1_{k-2}$. If $k=2$, let $w=0^{1 \times 1}$. Define $f_{\alpha, \beta}(\mathbf{y})$ to be the $n \times p$ matrix

$$
f_{\alpha, \beta}(\mathbf{y})=\left(\begin{array}{ccccccc}
0^{(k-1) \times 4} & 0^{(k-1) \times 4} & 0^{(k-1) \times 4} & N \beta & N \beta & w^{(k-1) \times 1} & 0^{(k-1) \times q} \\
\frac{1}{\sqrt{2}} A \alpha A^{T} & \frac{1}{\sqrt{2}} A \alpha A^{T} & \frac{1}{\sqrt{2}} A \alpha A^{T} & 0^{4 \times(k-2)} & 0^{4 \times(k-2)} & 0^{4 \times 1} & 0^{4 \times q} \\
-\frac{1}{\sqrt{2}} A \alpha A^{T}-\frac{1}{\sqrt{2}} A \alpha A^{T} & -\frac{1}{\sqrt{2}} A \alpha A^{T} & 0^{4 \times(k-2)} & 0^{4 \times(k-2)} & 0^{4 \times 1} & 0^{4 \times q} \\
\multicolumn{7}{c}{\sqrt{n \epsilon}} \\
\multicolumn{7}{c}{0^{(n-p-k-8) \times p}}
\end{array}\right) \in \mathcal{Y}
$$

(Here and below, omit any submatrices either of whose dimensions is 0 .) Note that the columns of $f_{\alpha, \beta}(\mathbf{y})$ are mean centered. Hence, the covariance matrix is given by the following:

$$
S\left[f_{\alpha, \beta}(\mathbf{y})\right]=\frac{1}{n}\left(\begin{array}{ccccccc}
A \alpha^{2} A^{T} & A \alpha^{2} A^{T} & A \alpha^{2} A^{T} & 0^{4 \times(k-2)} & 0^{4 \times(k-2)} & 0^{4 \times 1} & 0^{4 \times q} \\
A \alpha^{2} A^{T} & A \alpha^{2} A^{T} & A \alpha^{2} A^{T} & 0^{4 \times(k-2)} & 0^{4 \times(k-2)} & 0^{4 \times 1} & 0^{4 \times q} \\
A \alpha^{2} A^{T} & A \alpha^{2} A^{T} & A \alpha^{2} A^{T} & 0^{4 \times(k-2)} & 0^{4 \times(k-2)} & 0^{4 \times 1} & 0^{4 \times q} \\
0^{(k-2) \times 4} & 0^{(k-2) \times 4} & 0^{(k-2) \times 4} & \beta^{2} & \beta^{2} & b 1_{k-2} & 0^{(k-2) \times q} \\
0^{(k-2) \times 4} & 0^{(k-2) \times 4} & 0^{(k-2) \times 4} & \beta^{2} & \beta^{2} & b 1_{k-2} & 0^{(k-2) \times q} \\
0^{1 \times 4} & 0^{1 \times 4} & 0^{1 \times 4} & b 1_{k-2}^{T} & b 1_{k-2}^{T} & 1 & 0^{1 \times q} \\
0^{q \times 4} & 0^{q \times 4} & 0^{q \times 4} & 0^{q \times(k-2)} & 0^{q \times(k-2)} & 0^{q \times 1} & 0^{q \times q}
\end{array}\right)+\epsilon I_{p} .
$$


In particular, $S\left[f_{\alpha, \beta}(\mathbf{y})\right]$ is of full rank, $p$. Thus, $S\left[f_{\alpha, \beta}(\mathbf{y})\right]=K K^{T}+\epsilon I_{p}$, where

$$
\text { (7.12) } K_{\alpha, \beta}^{p \times k}=K_{\alpha, \beta}(\mathbf{y})
$$

$$
=\frac{1}{\sqrt{n}}\left(\begin{array}{ccccccc}
0^{(k-2) \times 4} & 0^{(k-2) \times 4} & 0^{(k-2) \times 4} & \beta & \beta & b \beta^{-1} 1_{k-2} & 0^{(k-2) \times q} \\
\alpha A^{T} & \alpha A^{T} & \alpha A^{T} & 0^{2 \times(k-2)} & 0^{2 \times(k-2)} & 0^{2 \times 1} & 0^{2 \times q}
\end{array}\right)^{T} .
$$

That is, $f_{\alpha, \beta}(\mathbf{y})$ is a perfect fit (section प1).

Let $\tilde{\mathcal{Y}} \subset \mathcal{Y}$ denote the set of data sets, $Y$, at which UMLE's exist and all UMLE's of $\Lambda$ at $Y$ have the same column space. By [14, Result 4.11, p. 147] and [2, Theorem $5.1]$

$$
\left(K_{\alpha, \beta}(\mathbf{y}), \epsilon I_{p}\right) \text { is a UMLE at } f_{\alpha, \beta}(\mathbf{y}) \text { and } f_{\alpha, \beta}(\mathbf{y}) \in \tilde{\mathcal{Y}}, \quad \mathbf{y} \in G^{2} .
$$

Now, $\Psi=\epsilon I_{p}$ and $\Lambda=K_{\alpha, \beta}$ do not satisfy (7.10). However, one can prove ([7])

$$
\begin{aligned}
& \text { If } \alpha_{1}>\alpha_{2}>2 \text { and } 1 / \sqrt{2}>\beta_{1}>\cdots>\beta_{k-2}>0, \\
& \text { then } f_{\alpha, \beta}(\mathbf{y}) \in \mathcal{Y}^{\prime} \text { for every } \mathbf{y} \in G^{2} .
\end{aligned}
$$

$f_{\alpha, \beta}$ is not necessarily continuous. However, let $\alpha_{1}=\alpha_{2}=3$ and $f=f_{\alpha, \beta}=f_{3 I_{2}, \beta}$. Then $A \alpha A^{T}=3 A(\mathbf{y}) A(\mathbf{y})^{T}=3 \Pi_{\mathbb{R}^{4}, \mathbf{y}}$. In particular, $A(\mathbf{y}) A(\mathbf{y})$ does not depend on which orthogonal basis of $\mathbf{y}$ is chosen as the columns of $A(\mathbf{y})$, and $\mathbf{y} \mapsto A(\mathbf{y}) A(\mathbf{y})^{T}$ is a continuous function of $\mathbf{y} \in G^{2}$. It follows that $f$ is continuous. Moreover, by (7.14), letting $\alpha \rightarrow 3 I_{2}$, we see that

$$
f(\mathbf{y}) \in \overline{\mathcal{Y}^{\prime}}, \quad \mathbf{y} \in G^{2} .
$$

Next, we show that

$$
\lim _{Y \rightarrow f(\mathbf{y}), Y \in \mathcal{Y}^{\prime}} \Phi(Y)=\text { the column space of } K .
$$

In particular, $f\left(G^{2}\right) \subset \mathcal{X}$, and $\Phi[f(\mathbf{y})]$ is just the column space of $K$. For suppose not. Then there exists a sequence $\left\{Y_{m}\right\} \subset \mathcal{Y}^{\prime}$ s.t. $Y_{m} \rightarrow f(\mathbf{y})$ but the column space, $\Phi\left(Y_{m}\right)$, of $\hat{\Lambda}_{m}$ is bounded away from that of $K$. Here, $\left(\hat{\Lambda}_{m}, \hat{\Psi}_{m}\right)$ is the MLE at $Y_{m}$. Claim: $\left\{\hat{\Psi}_{m}\right\}$ and $\left\{\hat{\Lambda}_{m}\right\}$ are bounded. Indeed, let

$$
\hat{\Sigma}_{m}=\hat{\Lambda}_{m} \hat{\Lambda}_{m}^{T}+\hat{\Psi}_{m} \text {. }
$$

Then $l\left(\hat{\Sigma}_{m}, S\left(Y_{m}\right)\right) \leq l\left(I_{p}, S\left(Y_{m}\right)\right)$. Since $S\left(Y_{m}\right) \rightarrow S[f(\mathbf{y})]$, it follows that $l\left(\hat{\Sigma}_{m}, S\left(Y_{m}\right)\right)$ is bounded above. Let $\Gamma_{m}=\hat{\Sigma}_{m}^{-1 / 2} S\left(Y_{m}\right) \hat{\Sigma}_{m}^{-1 / 2}$, where $\hat{\Sigma}_{m}^{-1 / 2} \hat{\Sigma}_{m}^{-1 / 2}=$ $\hat{\Sigma}_{m}^{-1}$. So

$$
a_{m} \equiv-\log \operatorname{det} S\left(Y_{m}\right)+l\left(\hat{\Sigma}_{m}, S\left(Y_{m}\right)\right)=\operatorname{trace} \Gamma_{m}-\log \operatorname{det} \Gamma_{m} .
$$

Now, $S\left(Y_{m}\right) \rightarrow S[f(\mathbf{y})]$, a nonsingular matrix. Therefore, $\log \operatorname{det} S\left(Y_{m}\right) \rightarrow$ $\log \operatorname{det} S[f(\mathbf{y})]$, which is finite. Therefore, $\left\{a_{m}\right\}$ is bounded above. Let $\nu_{1 m} \geq$ $\ldots \geq \nu_{p m} \geq 0$ be the eigenvalues of $\Gamma_{m}$. Then $a_{m}=\sum_{i=1}^{p}\left(\nu_{i m}-\log \nu_{i m}\right)$. Notice that, for $\nu>0$ we have $0<\nu-\log \nu$ and $\nu-\log \nu \rightarrow+\infty$ as $\nu \downarrow 0$ or $\nu \uparrow+\infty$. Hence, the $\nu_{i m}$ 's are bounded and bounded away from 0 . It follows that $\left\{\hat{\Sigma}_{m}\right\}$ is bounded. Since $\hat{\Psi}_{m}$ is nonnegative (by (7.10) ), it follows from (7.17) that $\left\{\hat{\Psi}_{m}\right\}$ and $\left\{\hat{\Lambda}_{m}\right\}$ are bounded, as claimed.

Therefore, by compactness, we may assume that $\left\{\hat{\Lambda}_{m}\right\}$ and $\left\{\hat{\Psi}_{m}\right\}$ converge to matrices $\hat{\Lambda}_{\infty}$ and $\hat{\Psi}_{\infty}$, respectively, and the column space of $\hat{\Lambda}_{\infty}$ is not the same as that of $K$. Now, $\Psi=\hat{\Psi}_{\infty}$ satisfies (7.10) and in fact $\left(\hat{\Lambda}_{\infty}, \hat{\Psi}_{\infty}\right)$ must be a UMLE at $f(\mathbf{y})$. For otherwise, for sufficiently large $m,\left(\hat{\Lambda}_{m}, \hat{\Psi}_{m}\right)$ cannot be a UMLE at 
$Y_{m}$. But this contradicts (7.11). On the other hand, by (7.13), $\left(\hat{\Lambda}_{\infty}, \hat{\Psi}_{\infty}\right)$ cannot be a UMLE because the column space of $\hat{\Lambda}_{\infty}$ is different from that of $K$. This contradiction establishes (7.16).

Let $\mathbf{x} \in G(k-2, p)$ be the row space of the matrix

$$
\left(0^{(k-2) \times 4} \quad 0^{(k-2) \times 4} \quad 0^{(k-2) \times 4} \quad \beta \quad \beta \quad b \beta^{-1} 1_{k-2} \quad 0^{(k-2) \times q}\right) .
$$

Let $\mathbf{g}_{2}: G^{2} \rightarrow G(2, p)$ take $\mathbf{y} \in G^{2}$ to the row space of

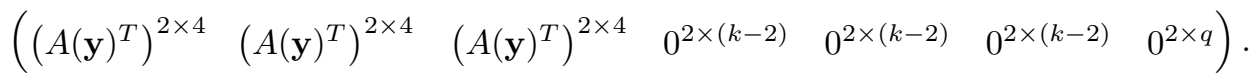

Let $\mathbf{g}: G^{2} \rightarrow G(k, p)$ take $\mathbf{y} \in G^{2}$ to $\mathbf{x} \oplus \mathbf{g}_{2}(\mathbf{y})$. Then $\mathbf{g}$ satisfies (7.5) (see (7.12)). Thus, maximum likelihood factor analysis (based on the covariance matrix) satisfies (7.5) (at least when (77.3) holds).

\section{Discussion AND CONCLUSIONS}

We have divided factor analysis into two components, the factor space and the collection, ordered or unordered, of lines in the factor space spanned by the individual loading vectors (the "factor lines"). We have seen that even where the factor space is stable, the factor lines can be unstable. Indeed, whenever the factor space function, $\Phi$, of a factor analysis method, $F$, exhibits sufficiently rich behavior, the factor lines of $F$ must be unstable at some data sets ("factor singularities") where the factor space is stable. The function, $\Phi$, will exhibit rich behavior, e.g., if $F$ often can find the right factor space at perfect fits (section (1).

Since good performance at perfect fits is a desirable quality in factor analysis methods, one wants the behavior of $\Phi$ to be rich. We have seen (section 7) that two popular factor analysis methods, principal components and maximum likelihood, recognize enough perfect fits so that the theory we have developed here applies to them, except perhaps if $n$ or $p$ is too small. An advantage of basing the theory on the behavior of the factor space function is that prima facie the factor space function is easier to study than the behavior of the complete factor analytic method. In particular, there is no need to be concerned about rotations.

Our methods are topological. That leads to very general results whose conclusions are difficult to escape. For example, consider the strategy

Given a data set, $Y$, choose a factor analytic method

that is continuous in a neighborhood of $Y$.

This sounds like a foolproof way to escape the singularity phenomenon, but it fails for at least two reasons. The first reason is that there may be data sets at which no factor analysis method is continuous, e.g., those whose covariance matrices have rank less than $k$. But the factor space function is also unlikely to be continuous at such degenerate data sets. Since we are concerned here only with data sets at which the factor space function is defined and continuous, we will ignore degenerate singularities.

A more fundamental difficulty with (8.1) is that any implementation of it will itself be a factor analysis method to which the theory we describe may apply.

Implementing (8.1) systematically amounts to defining a function that, given a data set, $Y$, selects a factor analysis method, $F_{Y}$, that is continuous in a neighborhood of $Y$. One then computes $F_{Y}(Y)$. But the net result of this procedure is a factor analysis method, $F^{*}$, that maps $Y$ to $F_{Y}(Y)$. Either the factor space 
function, $\Phi$, of $F^{*}$ behaves poorly, or the results of this paper apply to $F^{*}$ (at least if $k=2$ or 3 if $F^{*}$ is unordered). In the latter case, $F^{*}$ must have a factor singularity. A good place to look for singularities of $F^{*}$ is at those transitional data sets, $Y$, at which the factor analysis method, $F_{Y}$, changes. In any case, either $\Phi$ is impoverished or $F^{*}$ has at least one factor singularity.

We have shown that under general hypotheses a factor analytic method, $F$, will have factor singularities. In practice the set, $\mathcal{S}$, of factor singularities of $F$ almost certainly has Lebesgue measure 0 . So the probability of actually getting a singularity is 0 . However, singularities are important because, by definition (1.3), a factor analysis method will be unstable in the vicinity of its singularities, i.e., in a neighborhood of $\mathcal{S}$. It is reasonable to suppose that the probability of getting a data set in such a neighborhood is positive. The bigger $\mathcal{S}$ is, the larger should be the probability of getting data near $\mathcal{S}$. This raises the question, how big is $\mathcal{S}$ ? I conjecture that the codimension of $\mathcal{S}$ in $\mathcal{Y}$, i.e., the difference in dimensions ([9]) of $\mathcal{Y}$ and $\mathcal{S}$, is no greater than 2. (See [6, 8] for a discussion of this issue in the context of plane-fitting.)

\section{ACKNOWLEDGMENTS}

Michael Browne brought the paper Jennrich [13] to my attention. Robin Chapman showed me how to compute the fundamental group of $\mathbf{J}_{\{3\}}$. Martin Bendersky and John Stillwell provided other useful information. The presentation benefits from the comments of a referee.

\section{REFERENCES}

[1] Altmann, S. L. (1986) Rotations, Quaternions, and Double Groups. Clarendon Press, Oxford. MR 88e:20001

[2] Anderson, T. W. and Rubin, H. (1956) "Statistical inference in factor analysis," Proc. Third Berkeley Sympos. Math. Statist., J. Neyman, ed., Univ. of California Press, Berkeley, 111 150. MR 18:954f

[3] Boothby, W. M. (1975) An Introduction to Differentiable Manifolds and Riemannian Geometry. Academic Press, New York. MR 54:13956]

[4] Cayley, A. (1963) The Collected Mathematical Papers. Johnson Reprint Corporation, New York.

[5] Dayan, P. and Abbott, L. F. (2001) Theoretical Neuroscience: Computational and Mathematical Modeling of Neural Systems. MIT Press, Cambridge, MA.

[6] Ellis, S. P. (1995) "Dimension of the singular sets of plane-fitters," Annals of Statistics 23, 490-501. MR 97b:62083

[7] Ellis, S. P. (2002) "On the instability of factor analysis," unpublished manuscript.

[8] Ellis, S. P. (2002) "Fitting a line to three or four points on a plane," SIAM Review 44, 616-628.

[9] Falconer, K. (1990), Fractal Geometry: Mathematical Foundations and Applications. John Wiley \& Sons, New York. MR 92j:28008

[10] Golub, G. H. and Van Loan, C. F. (1996) Matrix Computations, Third Edition. Johns Hopkins University Press, Baltimore, MD. MR 97g:65006

[11] Greenberg, M. J. and Harper, J. R. (1981) Algebraic Topology: A First Course. AddisonWesley, Reading, MA. MR 83b:55001

[12] Harman, H. H. (1967) Modern Factor Analysis, Second Edition, Revised. University of Chicago Press, Chicago. MR 37:4909

[13] Jennrich, R. I. (1973) "On the stability of rotated factor loadings: The Wexler phenomenon," Br. J. Math. Statist. Psychol. 26, 167-176.

[14] Johnson, R. A. and Wichem, D. W. (1992) Applied Multivariate Statistical Analysis, Third Edition. Prentice Hall, Englewood Cliffs, NJ. MR 93c:62103 
[15] Lawley, D. N. and Maxwell, A. E. (1971) Factor Analysis as a Statistical Method. Butterworth \& Co., London. MR 49:8212

[16] Massey, W. S. (1967) Algebraic Topology: An Introduction. Harcourt, Brace \& World, Inc., New York. MR 35:2271

[17] Milnor, J. W. and Stasheff, J. D. (1974) Characteristic Classes. Annals of Mathematics Studies Number 76, Princeton University Press, Princeton, NJ. MR 55:13428

[18] Munkres, J. R. (1984) Elements of Algebraic Topology. Benjamin/Cummings, Menlo Park, CA. MR 85m:55001

[19] Psychological Corporation, The (1997) Wechsler Adult Intelligence Scale - Third Edition, Wechsler Memory Scale - Third Edition: Technical Manual. Harcourt Brace \& Co., New York.

[20] Spanier, E. H. (1966) Algebraic Topology. McGraw-Hill, New York. MR 35:1007

[21] Stillwell, J. (2001) "The story of the 120-cell," Notices of Amer. Math. Soc. 48, 17-24. MR 2001k:52019

[22] Stong, R. E. (1968) Notes on Cobordism Theory. Princeton University Press, Princeton, NJ. MR 40:2108

New York State Psychiatric Institute and Columbia University, Unit 42, NYSPI, 1051 Riverside Dr., New York, New York 10032

E-mail address: ellis@neuron.cpmc.columbia.edu 